\title{
Structure-based inhibitor screening of natural products against NSP15 of SARS-CoV-2 revealed thymopentin and oleuropein as potent inhibitors
}

\author{
Ramachandran Vijayan ${ }^{1} \cdot$ Samudrala Gourinath $^{1}$ (I)
}

Received: 12 December 2020 / Revised: 26 February 2021 / Accepted: 9 March 2021 / Published online: 23 March 2021

( ) The Author(s), under exclusive licence to Springer Nature Singapore Pte Ltd. 2021

\begin{abstract}
Coronaviruses are enveloped, non-segmented positive-sense RNA viruses with the largest genome among RNA viruses. Their genome contains a large replicase ORF which encodes nonstructural proteins (NSPs), structural, and accessory genes. NSP15 is a nidoviral RNA uridylate-specific endoribonuclease (NendoU) with C-terminal catalytic domain. The endoribonuclease activity of NSP15 interferes with the innate immune response of the host. Here, we screened Selleckchem Natural product database of the compounds against NSP15, and we found that thymopentin and oleuropein displayed highest binding energies. The binding of these molecules was further validated by molecular dynamic simulations that revealed them as very stable complexes. These drugs might serve as effective counter molecules in the reduction of virulence of this virus; may be more effective if treated in combination with replicase inhibitors. Future validation of both these inhibitors is worth the consideration for patients being treated for COVID-19.
\end{abstract}

Keywords Drug designing · Virtual screening · Severe acute respiratory syndrome coronavirus 2 (SARS-CoV-2) · Natural products

\section{Introduction}

Severe acute respiratory syndrome coronavirus 2 (SARS$\mathrm{CoV}-2$ ) is known to cause the novel coronavirus disease of 2019 (COVID-19) (Cavanagh 1997). SARS-CoV-2 causes a wide array of respiratory, gastrointestinal, and neurological diseases in humans (Hilgenfeld 2013; Fehr and Perlman 2017; Bartlam and Rao 2005). Coronavirus has the largest genomes among the known RNA viruses, ranging $~ 30 \mathrm{~kb}$ (Snijder et al. 2003), which encodes structural proteins, S (spike), E (envelope), M (membrane), (Sawicki 2005; Gorbalenya et al. 2006), and non-structural N (nucleocapsid) proteins (Prentice et al. 2004; Zhang et al. 2018). The RNA-dependent RNA polymerase (NSP12) and proteases are very crucial for the survival and the replication of this virus (Kumar et al. 2020), and several groups around the world are targeting these proteins (Mohammad et al. 2020;

Samudrala Gourinath

sgourinath@mail.jnu.ac.in

1 School of Life Sciences, Jawaharlal Nehru University, New Delhi 110067, India
Khan et al. 2020). Non-structural protein 15 (NSP15) is an endoribonuclease that cleaves $3^{\prime}$ of uridylate through a ribonuclease A (RNase A) action (Ricagno et al. 2006; Bhardwaj et al. 2008). Though it is not required for viral replication, but it mediates evasion of host dsRNA sensors (Kindler et al. 2017; Deng 2018). NSP15 (NendoU) is a $\mathrm{Mn}^{2+}$-dependent RNA endonuclease, that generates $2^{\prime}-3^{\prime}$-cyclic phosphates (Ricagno et al. 2006). Nsp15 is responsible for the suppression of type I IFN (IFN- $\alpha / \beta)$-associated innate immune response by infecting the macrophages (Deng et al. 2017). NSP15 degrades viral RNA to prevent itself from the host defenses (Ricagno et al. 2006).

The structure of SARS-CoV-2 NSP15 protein is very similar to other coronavirus NSP15 monomers, consisting of mainly three regions: The $\mathrm{N}$-terminal domain, a subsequent middle domain, and a catalytic C-terminal nidoviral RNA uridylate-specific endoribonuclease domain. The active-site residues of NSP15, His-262, His-277, and Lys-317 are conserved across the entire endoribonuclease family and play an important role in enzyme catalysis (Deng 2018). The NSP15 mutant viruses cause apoptotic cell death with significant reduction of replication in macrophages (Kang et al. 2007; Posthuma et al. 2006). 
SARS-CoV-2 has infected more than 100 million people and caused over 2470,000 deaths worldwide (https:// www.who.int) (data as of 24 February 2021). The rapidly increasing numbers of the infected patients prompted the World Health Organization to declare a state of global health emergency to coordinate the scientific and medical efforts to expedite the development of a cure for the patients.

NSP15 is important for disease progression and virulence, and thus it is a potential target for drugs. Till date, no targeted treatment molecule has been developed to prevent the COVID-19 infection. Recently, the in silico studies have reported the possible inhibitors against NSP15 (Chikhale et al. 2020; Sinha et al. 2020a, b; Chandra et al. 2020; Quimque et al. 2020). Remedesivir and Favipiravir are the latest approved drugs for the treatment of COVID-19, which target and inhibit the replicase (Bimonte et al. 2020; Jean and Hsueh 2020). However, although, these drugs are able to reduce the recovery time, they are not able to help the critically ill patients. Further, these drugs cannot control the outbreak of the novel coronavirus and the subsequent pandemic (Slam et al. 2020). To design specific inhibitors against the non-structural protein 15 (NSP15), libraries of Selleckchem Natural Product (https://www.selleckchem.com/scree ning/natural-product-library.html) were chosen for virtual screening. Top ten compounds were selected based on their binding affinities. Further, molecular dynamic simulation was used to analyze the stability and the inter-molecular interactions between the NSP15 and the lead compounds. The top-ranked molecule with the highest binding affinity was thymopentin, which is an already FDA-approved drug, generally given to improve the immunity. Thus, this repurposed molecule could inhibit NSP15 to decrease the viral virulence and improve the host immunity.

\section{Materials and methods}

The crystal structure of the non-structural protein 15 (NSP15) was retrieved from the RCSB Protein Data Bank (entry code 6W01) and was used as target for our modeling studies. Starting structure of NSP15 for docking studies was prepared with Protein Preparation Wizard (Schrödinger, LLC 2020a, b). The process adds hydrogen, neutralizes appropriate amino acid chains, and relieves steric clashes. Also, it performs a series of restrained, partial minimizations on the co-crystallized structure, each of which employs a limited number of minimization steps. It is not intended to minimize the system completely. In our study, the minimization (OPLS 2003 force field) (Schrödinger, LLC 2020a, b) was stopped when RMSD of the non-hydrogen atoms reached $0.30 \AA$, which is the default specified limit.

\section{Preparation of ligand library}

Selleckchem Natural product libraries (https://www.selle ckchem.com/screening/natural-product-library.html) consist of FDA approved (2863 compounds), FDA approved and passed Phase I library (3176 compounds), Pre-clinical/ clinical compound library (2973 compounds), Bioactive compound I library (7749 compounds), Bioactive compound II library (5309 compounds), and natural compounds (2617 compounds). In totality, 24,678 compounds were chosen for virtual screening. Approved drugs, investigational drugs and natural products have been used to meet the urgent need of drug repurposing for the treatment of COVID-19. Libraries were prepared by energy minimization of 100 steps with the Ligprep module of Schrodinger (Epik LigPrep, Schrödinger, LLC 2020a, b).

\section{Docking-based virtual screening}

Experimental studies on NSP15 have revealed that His235, His-250, and Lys-290 (Ricagno et al. 2006) are the critical residues for catalysis that are located in the C-terminal domain of the enzyme. Docking studies were performed using GOLD (Jones et al. 1997), and the protein was kept as a rigid molecule, and the number of Genetic Algorithm (GA) runs was set to 10 runs per ligand with the default search algorithm parameters similar to our earlier reports (Vijayan and Mallick 2007; Eniyan et al. 2018; Chadha et al. 2018; Vijayan and Manohayan 2015; Mahendran and Vijayan 2018). GOLD score was then used as the final scoring method.

To estimate the accuracy of binding affinity of GLIDE (Friesner et al. 2006), MOE docking (Eniyan et al. 2018) and Auto-dock (Forli et al. 2016) were used for cross-docking analysis. In the GLIDE-docking (Friesner et al. 2006), the prepared structure was used to generate the receptor grid and no scaling was done for the Van der Waals radii of nonpolar receptor atoms. An enclosing box was used as the docking space, centered on the His-235, His-250, and Lys-290, catalytic triad using the crystallographic position as their reference; the ligand diameter midpoint box was set to the default value (10 ̊). Docking experiments were performed using 0.8 to scale the Van der Waals radii of the nonpolar ligand atoms with a charge cutoff of 0.15 . Poses were discarded as duplicates if the rms deviations in the ligand (all atoms) were less than $0.5 \AA$ and maximum atomic displacement was less than $1.3 \AA$. At most, ten poses per ligand were retained. GlideScore XP (Friesner et al. 2006) was used as the scoring method to finalize the screening.

For MOE docking the protein was kept as a rigid entity, and a maximum of ten conformations for each ligand was 
taken using the default parameters of MOE with triangle matcher placement. The top ranked conformations of NSP15 with the lead-like compounds docked conformations was stored. On the basis of MOE scoring \{Generalized-Born Volume Integral/Weighted Surface Area (GBVI/WSA) \}, binding free energy calculation in the $\mathrm{S}$ field denotes the score. The GBVI/WSA is a scoring function that estimates the free energy of binding of the ligand for a given pose as described before (Eniyan et al. 2018). For all scoring functions, lower scores indicate the more favorable poses. Top ranked docked conformations of the lead-like compounds were selected for further evaluation.

In the AutoDock 4.2 (Forli et al. 2016), empirical free energy function and the Lamarckian Genetic Algorithm were used for all the docking calculations and the AutoDockTools (ADT) package (Forli et al. 2016) was used to generate input files and to analyse the results. NSP15 was set to be rigid and all the torsional bonds were kept free during the docking process in the small molecules. The solvent molecules were not considered during the docking process. A cubic grid box of $60 \AA$ size $(\times 62.520, y 73.546, \mathrm{z} 29.278)$ with a spacing of $0.375 \AA$ was used and corresponding grid maps were created. The ten best solutions based on the docking scores were retained for further investigations. XSCORE (Renxiao 2002) has been used to estimate the binding affinity of the molecules. Interactions between the protein and the compounds were calculated using the Discovery Studio program (Dassault Systems 2016).

\section{Molecular dynamic simulations}

Molecular dynamics (MD) simulations were conducted for the modeled systems in an explicit solvent using the Groningen Machine for Chemical Simulations (GROMACS) package according to our previously described methodology (Vijayan et al. 2016; Waziri et al. 2019; Sharma et al. 2018; Dharavath et al. 2020). Molecular dynamics simulations were performed for the top five screened compounds (Thymopentin, Ginsenoside, Oleuropein, Akebia Saponin D, and Keampferitrin), using the GROMOS 53A6 force field (Berk and Erik 2008) to understand the dynamic behaviour, structural stability, residual fluctuation, and compactness of the protein in its complex form. Both systems were solvated using TIP3P water models with minimum distances of $10 \AA$ between the complex surfaces and the box edge in separate cubic boxes. Both systems were neutralized by the addition of physiological ionic strengths $(0.15 \mathrm{M})$ of counter ions and were energy minimized using the steepest descent algorithm with restraints of a $1000 \mathrm{~kJ} \mathrm{~mol}^{-1} \mathrm{~nm}^{-1}$ force constant up to a maximum of 5000 steps to remove steric clashes and bad Van der Waals contacts between the side chain and the main chain atoms. The LINCS algorithm was employed to constrain all the bonds, whereas the SETTLE algorithm was employed to construct the geometry of water molecules. The particle mesh Ewald (PME) method was used for long-range electrostatic interactions. The energy minimized models were subjected to equilibration using the NVT and NPT ensemble. Initially, the first round of equilibration involved an NVT simulation of $500 \mathrm{ps}$ by applying the velocity-rescaling thermostat, whereas the second round involved a NVT simulation of 500 ps using the Parrinello-Rahman barostat. After equilibration, both systems were subjected to a $100 \mathrm{~ns}$ long MD simulation. All the methods employed for MD simulation were adopted from earlier works (Vijayan et al. 2016; Waziri et al. 2019; Sharma et al. 2018). The time-dependent behaviour of both systems including the root mean square deviation (RMSD), radius of gyration ( $\mathrm{Rg}$ ), and root mean square fluctuation (RMSF) of $\mathrm{C} \alpha$-atoms was performed using tools available on GROMACS. Two-dimensional (2D) plots were generated using the Grace-5.1.23 program (http:// plasma-gate.weizmann.ac.il/Grace/). Molecular visualizations were performed using Biovia Discovery Studio Visualizer (Accelrys Inc., USA).

\section{Results}

Superposition of NSP15 with all other available coronavirus homologues reveals structural conservation amongst these proteins. The structure of the SARS-CoV-2 yielded a RMSD of $0.4 \AA$ and $0.9 \AA$ with SARS-CoV, and MurineCoV NSP15, respectively (Fig. 1). Comparison of all the three structures showed some deviations in the active site residues as marked in Fig. 2. These conformational changes at the active site of NSP15 need to be considered carefully during the inhibitor screening.

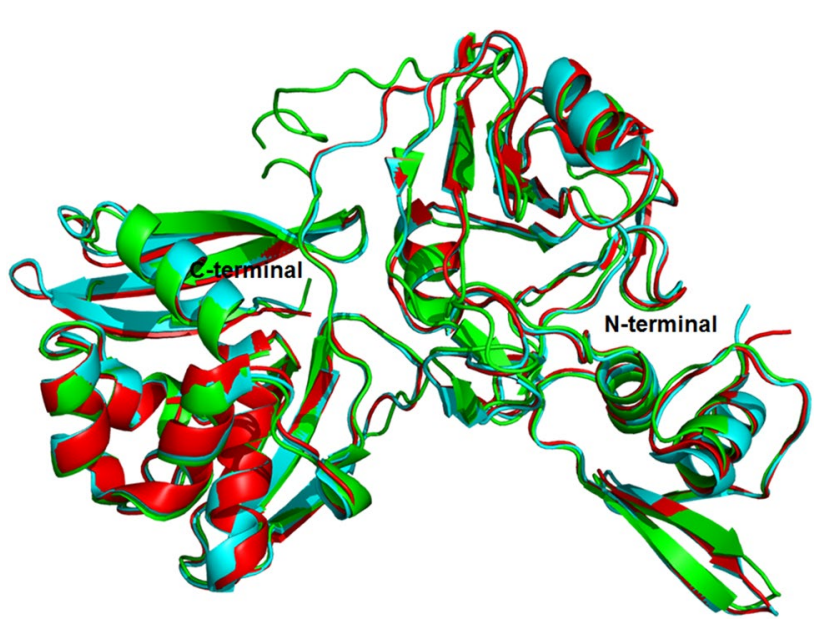

Fig. 1 Superimposition of NSP15 from different coronaviruses (CoVs). Superposition of structures at the active site from SARSCoV-2 (red), SARS-CoV (green) and Murine-CoV (cyan) showed that the structures are very similar to each other 


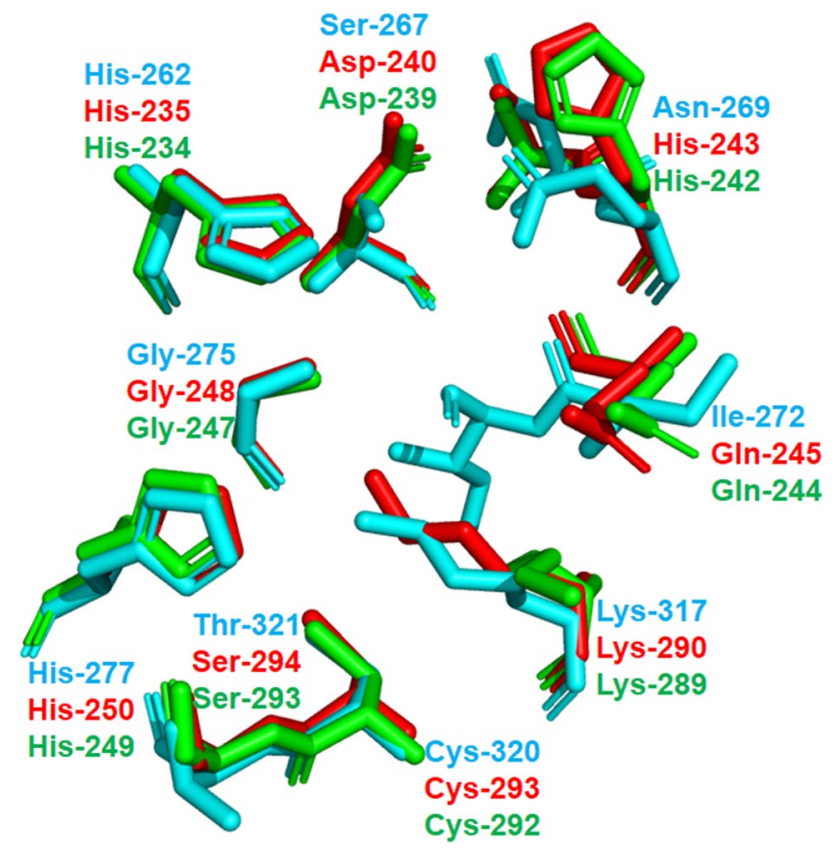

Fig. 2 Analysis of active site of NSP15: Residues at the drug-binding region of SARS-CoV2 (red color), SAR-CoV (green color) and murine-CoV (cyan color) are shown

NSP15 is a known antiviral drug target, and its active site constitutes the catalytic triad His-235, His-250, and Lys-290 (Fig. 2). To find drugs that could potentially inhibit NSP15, the crystal structure of NSP15 endoribonuclease from SARS-CoV-2 in complex with citrate at $1.9 \AA$ (PDB ID: 6W01) was used as the target to perform virtual screening with the Selleckchem Natural Product database containing 24,678 small molecules as potential binders for this active site. His-235, His-250, Lys-290, Thr-341, Tyr-343, and Ser294 reside at the active site.

\section{Structure-based screening}

GOLD (Jones et al. 1997) was used to perform virtual screening. Further, multiple docking algorithms using GLIDE (Friesner et al. 2006), Auto-dock (Forli et al. 2016), and MOE (Eniyan et al. 2018) docking ensured the accurate prediction of the binding affinities. These docking algorithms are most commonly used to estimate the binding affinity and binding pose of the inhibitor and the enzyme. The identification of the inhibitors for various targets using these docking and virtual screening algorithms obtained high successful rate based on our previous studies (Vijayan et al. 2016; Dharavath et al. 2020; Waziri et al. 2019). Therefore, we used all of the above-mentioned software to dock the Selleckchem Natural Product database (https://www. selleckchem.com/screening/natural-product-library.html) of 24,678 small molecules against NSP15 to evaluate their binding affinities. Also, we docked the previously reported ligands (Chikhale et al. 2020; Sinha et al. 2020a, b; Chandra et al. 2020, Quimque et al. 2020; Cinatl et al. 2003, Curreli and Flore 2005), Remedesvir (Bimonte et al. 2020; Jean and Hsueh 2020) and Lopinavir (Bimonte et al. 2020, Jean and Hsueh 2020) as reference molecules.

The top-ranked docked molecules were sorted according to their GOLD score (Jones et al. 1997), GLIDE score (Friesner et al. 2006), Auto-dock free energy of binding (Forli et al. 2016), MOE score (Eniyan et al. 2018) and XSCORE (Renxiao 2002) (Table 1). Mainly the mode of binding and their potential interactions with the active site residues of NSP15, and the estimated binding affinities were the major factors considered while shortlisting the compounds (Table 1) (Figs. 3, 4).

\section{Insights into binding}

We selected the top ten compounds (Fig. 5) for further analysis since these drug compounds yielded higher binding affinities (Table 1). The top ten compounds were found to fit tightly into the hydrophobic binding pocket and in close interaction with the catalytic triad. Among these compounds, five compounds showed higher binding affinities compared to the reported compounds for NSP15 (Table S1) (Figure S1). The top ranked molecule Thymopentin makes extensive interactions with the active site and the surrounding residues. Thymopentin's benzene moiety, enolizable ketone, and carboxylate groups are attached in the hydrophobic pocket of the binding site. Eight residues, Gly-247, Val-292, Ser-294, Val-315, Met-331, Lys-335, Thr-341, Pro-344 form Van der Waals interactions. Fourteen residues, His-235, Asp-240, Ser-242, His-243, Gln-245, Leu-246, Gly-248, His-250, Asn-278, Lys-290, Glu-340, Thr-343, Lys345, Leu-346 are forming electrostatic interactions with thymopentin. Asn278 and Leu-346 make two hydrogen bonds with the benzene-OH group of thymopentin. Thr343, Gln245, Gly-248, and Lys-290 form single hydrogen bond with the enolizable ketone. His 235 forms single hydrogen bond and Asp-240 make two hydrogen bonds with carboxylate group of thymopentin. The second ranked molecule Oleuropein interacts electrostatically with 11 residues His-235, Gln-245, Leu-246, Gly-248, His-250, Asn-278, Lys-290, Val-292, Cys-293, Ser-294, and Glu-340. Five residues, Gly-247, Trp-333, Lys-335, Thr-341, Pro-344, and Lys-345 make Van der Waals interactions. Glu-340 makes two hydrogen bonds with benzene-OH group of Oleuropein. Gly-248 and Lys-290 forms single hydrogen with 3-methylene-4H-pyran of Oleuropein. The toxicity analysis does not indicate any adverse effects for the top compounds (Table S2) and can be thus taken forward for further studies. 


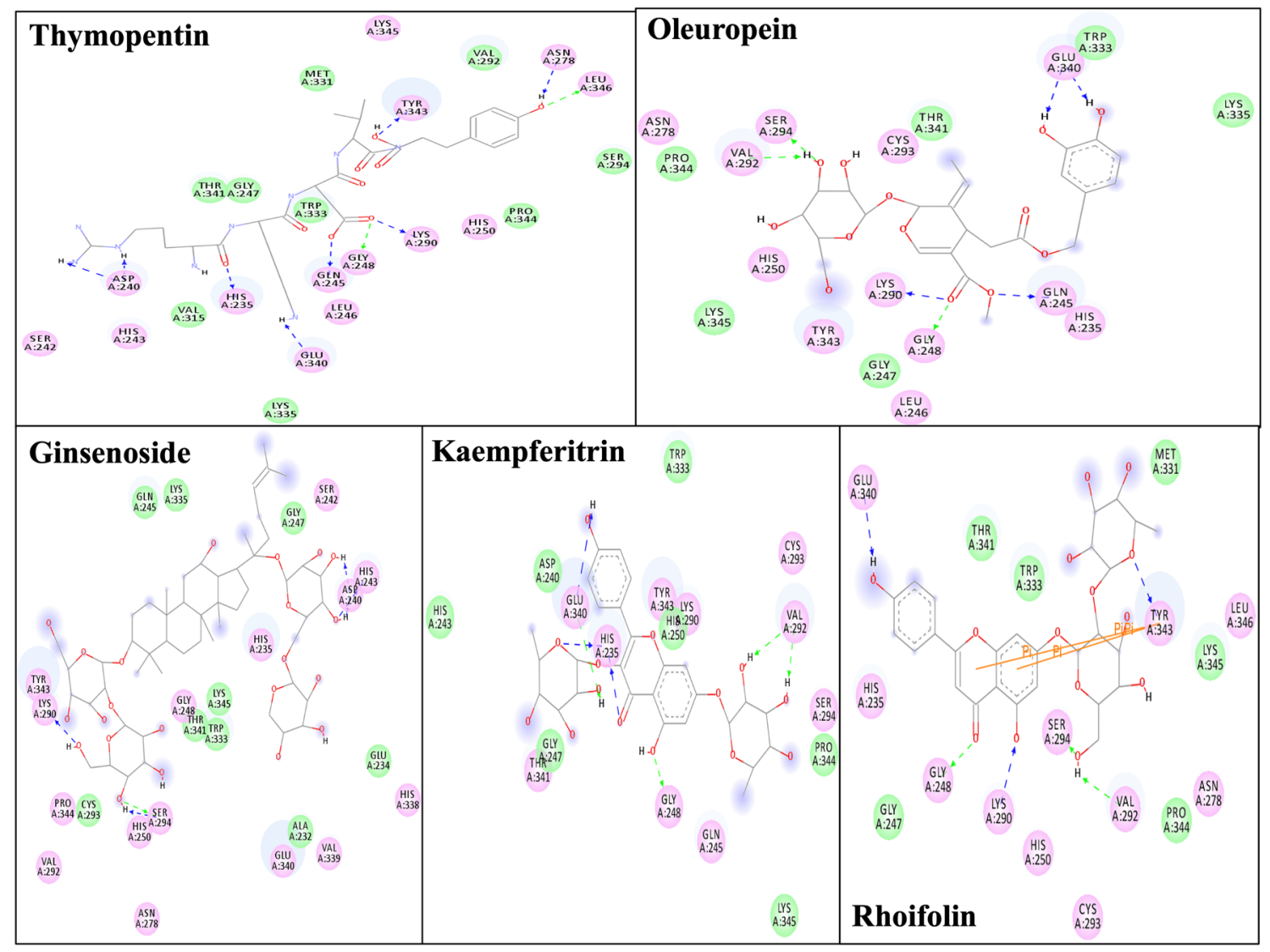

Fig. 3 Modes of binding and its interactions of the top five-inhibitor leads with NSP15

After the docking studies, the molecular dynamic simulation was performed for the top five screened compounds (Thymopentin, Ginsenoside, Oleuropein, Akebia Saponin D, and Kaempferitrin), to understand the binding stability of the docked complexes. The simulations were performed for a $100 \mathrm{~ns}$ to analyze the conformational stability of these complexes.

Molecular dynamic simulation is considered to be a reliable approach with greater insight into the dynamic behavior of proteins and that of the ligand conformations as observed in our earlier studies (Dharavath et al. 2020; Waziri et al. 2019; Sharma et al. 2018). The simulations provide detailed information to better understand the motions of the individual atoms as a function of time and properties of the molecules. We carried out a $100 \mathrm{~ns}$ MD simulation to accurately predict the binding stability on the identified compounds against NSP15 as explained in the "Methods". We have studied the root mean square deviation (RMSD), root mean square fluctuation (RMSF), radius of gyration ( $R g$ ), solvent accessible surface area (SASA), and hydrogen bonding interactions $(\mathrm{NH})$ between the NSP15 alone as well as in complex with the top five inhibitors. A total of six independent $100 \mathrm{~ns}$ simulations were carried out.
According to the RMSD (root-mean-square deviation) calculation, shown in Fig. 5, it can be seen that all the complexes tend to achieve equilibrium after $10 \mathrm{~ns}$ and lead to a stable trajectory throughout the simulation. We found that Ginsenoside, Kaempferitrin and Akebia saponin D complexes with NSP15 tend to reach a higher equilibrium compared to the native, and remained distinguished throughout the simulation, resulting in the RMSD of $0.1-0.75 \mathrm{~nm}$. The RMSD trajectories for the Thymopentin and Oleuropein complexes with NSP15 observed a lower equilibrium compared to the native with minor differences in their trajectory that leads to a stable equilibrium through the end of the simulation, suggesting that the complexes stabilized themselves (Fig. 6a). The higher RMSD obtained for all the complexes were limited to $0.75 \mathrm{~nm}$ that demonstrates the stable trajectories and provided us with an appropriate basis for further investigation. (Fig. 6a).

To observe the ligand induced conformational changes of the protein, we calculated the C-RMSF to observe the overall flexibility of the atomic positions in the trajectory for the native and the protein-ligand complexes (Fig. 6b). The flexibility of the residues in each of the protein-ligand was analyzed by means of RMSF, where the higher RMSF 


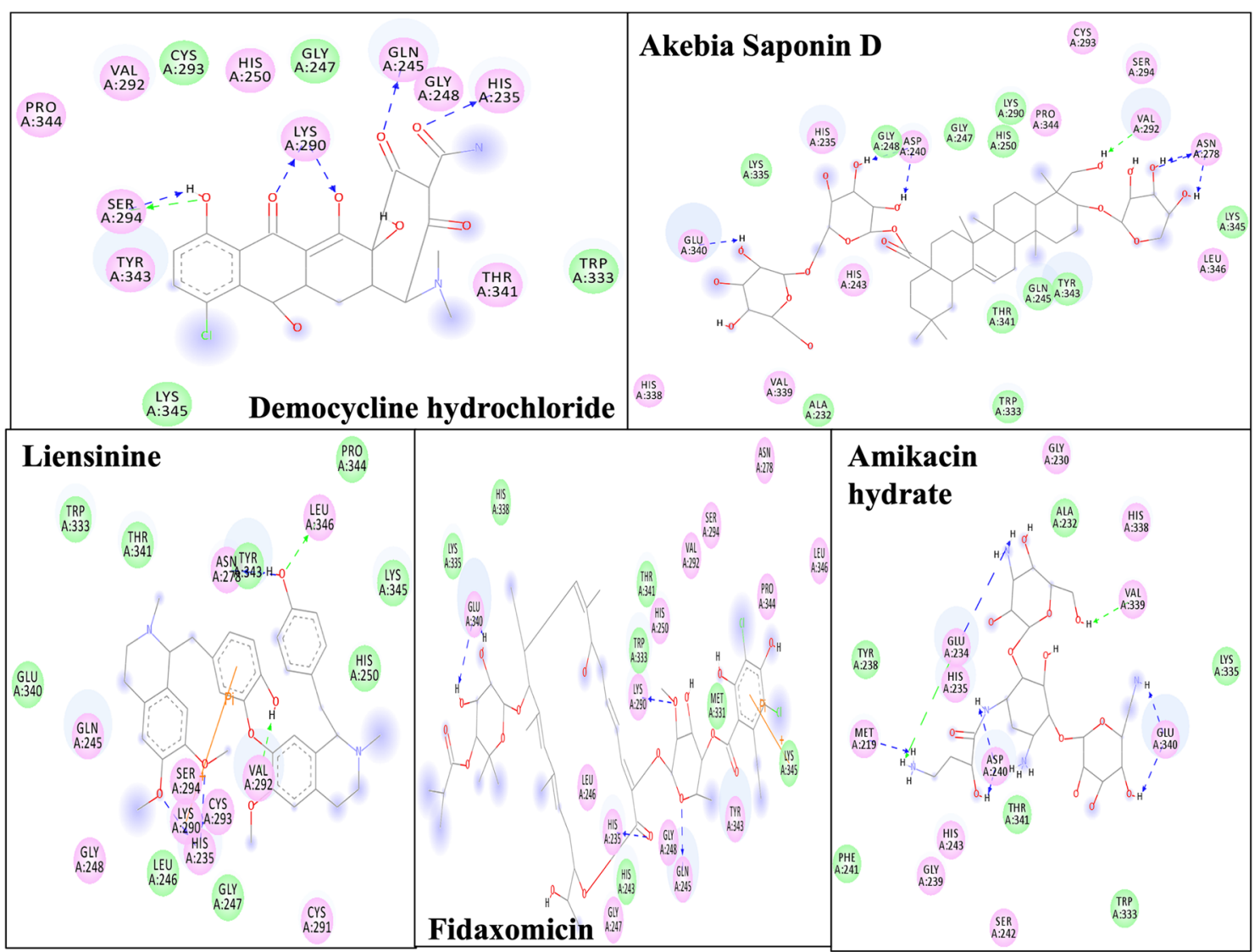

Fig. 4 Modes of binding and its interactions of the next five-inhibitor leads with NSP15

Fig. 5 The chemical structure of the top scoring compounds identified from virtual screening

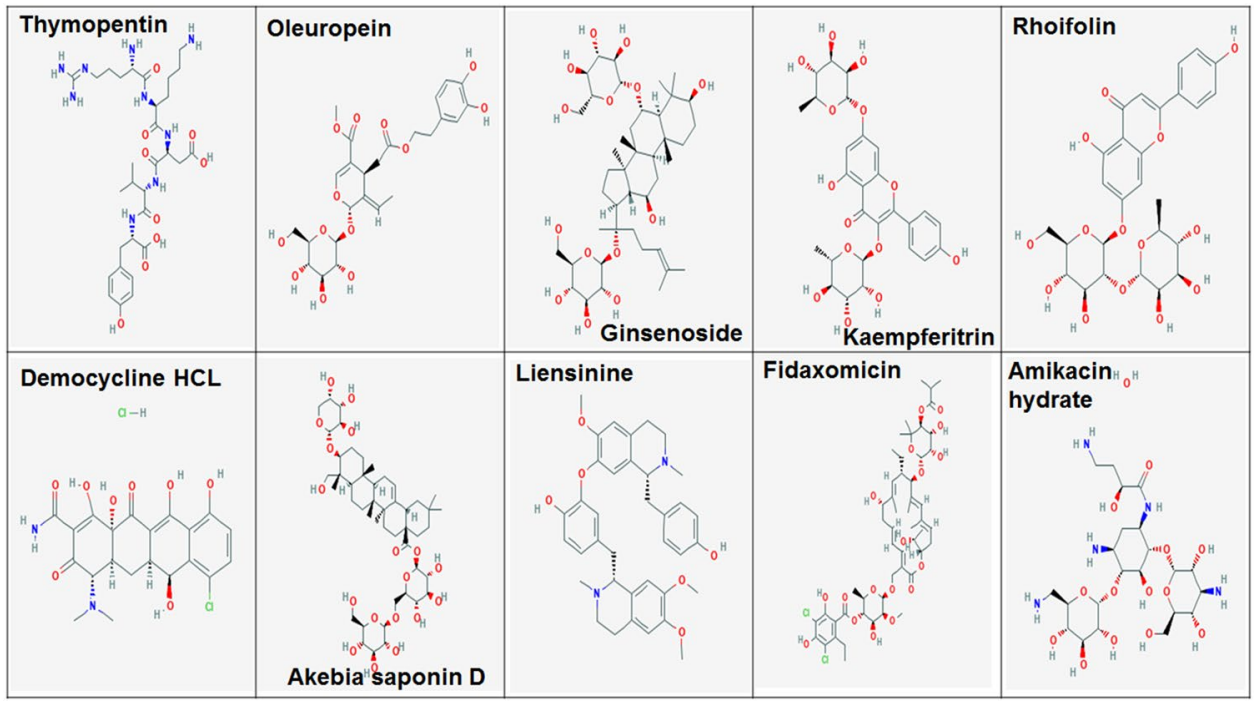

value describes the higher flexibility. According to Fig. 5b, Akebia Saponin D and Kaempferitrin produced higher fluctuations in the residue positions 40, 90, 110, 200, 250, $275,290,310$, and 320 during the simulation, while low fluctuations were observed for Ginsenoside, thymopentin,
Oleuropein, except at the 150th residue position. Overall, the RMSF result showed that the ginsenoside, thymopentin, and oleuropein complexes were more stable than the Akebia saponin D and kaempferitrin complexes (Fig. 6b). 
Table 1 Screening of NSP15 with the top-ten inhibitors and their estimated binding affinities

\begin{tabular}{lllllccc}
\hline No & Ligands & GOLD score & $\begin{array}{l}\text { GLIDE score } \\
\mathrm{kcal} / \mathrm{mol}\end{array}$ & $\begin{array}{l}\text { Auto-dock } \\
\mathrm{kcal} / \mathrm{mol}\end{array}$ & $\begin{array}{l}\text { MOE } \\
\mathrm{kcal} / \mathrm{mol}\end{array}$ & $\begin{array}{l}\text { X-Score } \\
\mathrm{kcal} / \mathrm{mol}\end{array}$ \\
\hline 1 & Thymopentin & 86.59 & -11.030741 & -9.7 & -12.811 & -9.63 & 8.49 \\
2 & Oleuropein & 84.69 & -10.960358 & -8.5 & -17.1605 & -8.70 & 7.50 \\
3 & Ginsenoside & 83.83 & -9.856758 & -8.0 & -14.4559 & -8.92 & 6.54 \\
4 & Kaempferitrin & 83.32 & -9.17588 & -7.0 & -17.3375 & -9.04 & 7.63 \\
5 & Rhoifolin & 82.14 & -9.231593 & -6.5 & -12.1433 & -8.43 & 7.18 \\
6 & Demeclocyclin hydrochloride & 81.71 & -8.553255 & -8.1 & -18.0979 & -8.15 \\
7 & Akebia Saponin D & 80.17 & -8.173799 & -7.5 & -12.3011 & -8.89 \\
8 & Liensinine & 79.31 & -7.752487 & -7.7 & -12.4259 & -8.10 \\
9 & Fidaxomicin & 79.14 & -10.166778 & -7.6 & -15.6436 & -8.58 & 7.25 \\
10 & Amikacin hydrate & 73.85 & -8.754372 & -7.0 & -12.7334 & -7.80 \\
11 & Lopinavir & - & - & - & -5.0902 & - \\
12 & Remedesivir & - & - & - & -5.5850 & - \\
\hline
\end{tabular}

A

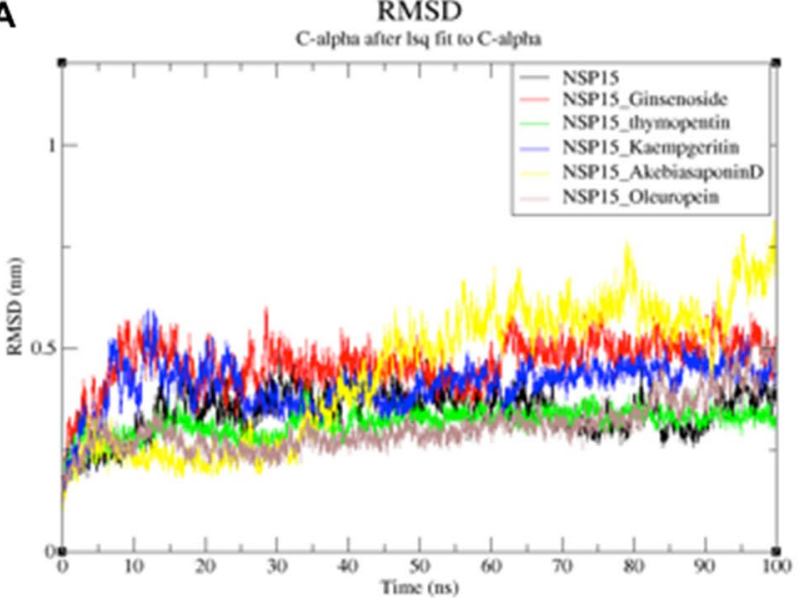

C Radius of gyration (total and around axes)

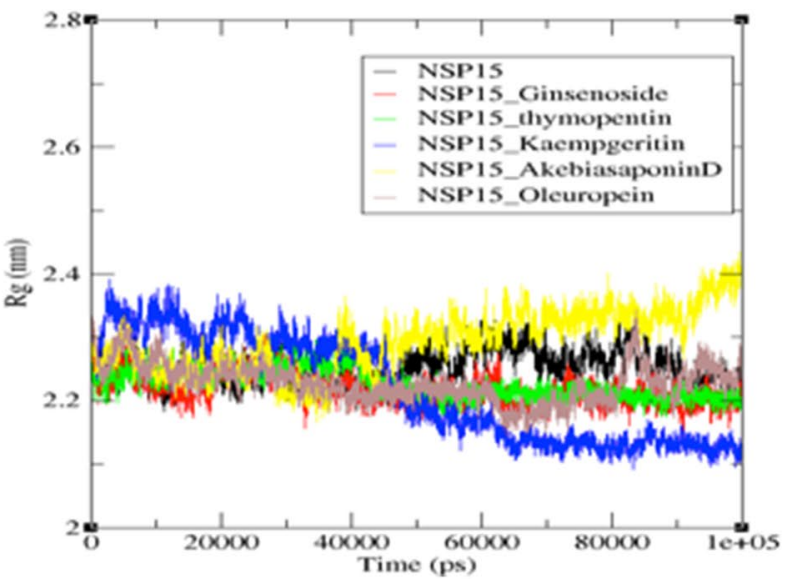

Fig. 6 MD simulation results for NSP15 with ligands. a RMSD analysis for the model system NSP15 protein alone and protein in complex with hits compounds. b Radius of gyration plot for NSP15 and
B RMS fluctuation

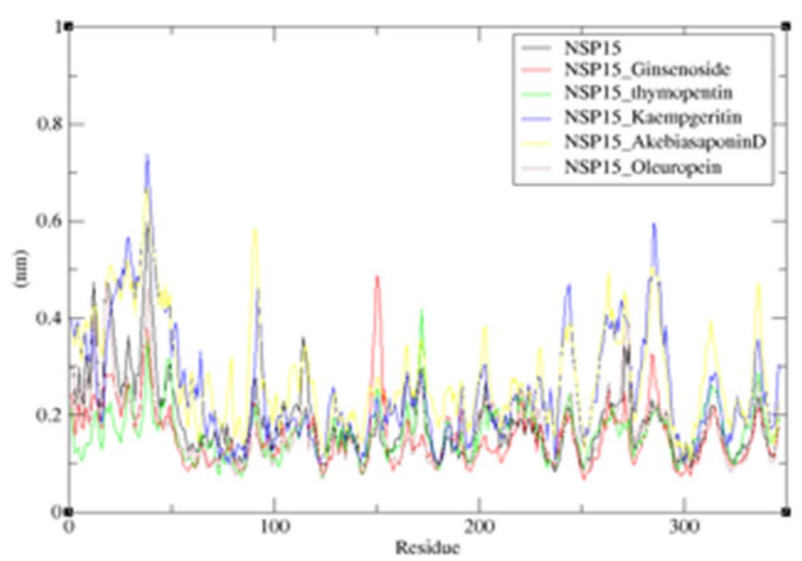

D Solvent Accessible Surface

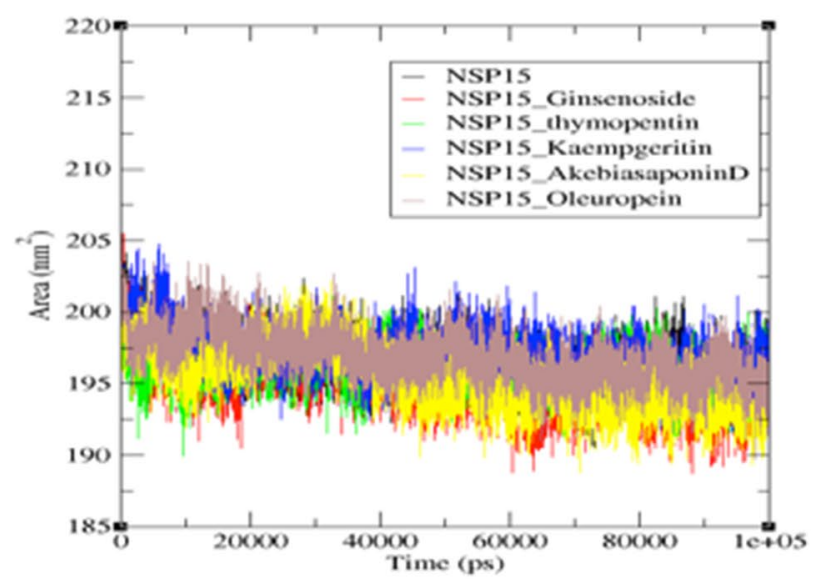

top 5 compounds complexes. c RMSF plot for NSP15 and top 5 hit compounds complexes. d SASA plot for NSP15 and top 5 hit compounds complexes 
The $\mathrm{Rg}$ (radius of gyration) is used to calculate the compactness of a protein (Fig. 5c). Rg is the root mean square distance of a particular atom or group of atoms with its center of mass. The overall NSP15 structure at various time points during the trajectory can be analyzed for the compactness, shape, and folding in the Rg plot (Fig. 5c). Akebia saponin D-NSP15 complex showed a higher deviation with a Rg score of $2.4 \mathrm{~nm}$. All the other compounds, ginsenoside, thymopentin, oleuropein, and kaempferitrin had an aggregate $\mathrm{Rg}$ score of $2.2 \mathrm{~nm}$ in a decreasing trend followed by stabilization after 40 ns towards the end of the simulation (Fig. 6c).

We have measured the compactness of the hydrophobic core by analyzing the changes in SASA. As shown in Fig. 5d, an increase of the SASA values were observed for Akebia saponin D and kaempferitrin after $10 \mathrm{~ns}$ of simulation time maintained till the end, while the ginsenoside, thymopentin, and oleuropein complexes showed stable conformation throughout the simulations. As higher SASA value leads to an increased exposure, there was loss of hydrophobic contact between the protein-ligand complexes (Fig. 6d).

Finally, the number of hydrogen bonds for each of the protein complex was calculated during the simulation. An increase in the number of hydrogen bonds and Van der Waals and electrostatic interactions were observed for ginsenoside, thymopentin, oleuropein, while Akebia saponin D and kaempferitrin formed lesser interactions (Figs. 3, 4). The number of hydrogen bonds formed between NSP15 in complex with the top five inhibitors before and after simulations were also calculated (Fig. S3). From our analysis, it is revealed that At $0 \mathrm{~ns}$, the NSP15-Akebia saponin D and NSP15-Ginsenoside forms with an average of $\sim 11$ and $~ 10$ NH bonds (Fig. S3). While the other complexes, NSP15Thymopentin, NSP15-Oleuropein and NSP15-Kaempferitrin exhibited a fewer number of intermolecular hydrogen bonds, with an average of $\sim 8$ (Fig. S3). After $70 \mathrm{~ns}$, all the complexes throughout the simulation reached stable number of hydrogen bonds with an average of $\sim 8$ (Fig. S3).

\section{Discussion}

Thymopentin and oleuropein exhibited the highly ranked docking score of -12.811 , and $-17.1605 \mathrm{kcal} / \mathrm{mol}$, respectively (Table 1) (Figs. 3, 4). We have compared docking results of reported SARS-CoV-2 inhibitors (Fig. S1 and Fig. S2), withanoside X, saikosaponins, glycyrrhizic acid, lopinavir, ribavirin, glisoxepide, idarubicin, quinadoline $\mathrm{B}$, and remedesivir and the analysis showed that the binding affinities were found to be significantly lower as compared to our identified compounds, thymopentin and oleuropein. The top ten compounds were found to tightly bind the hydrophobic binding pocket and in close interaction with the catalytic triad. The in silico toxicity analysis does not indicate any adverse effects for the top compounds (Table S2).

The top-ranked compound obtained from the virtual screening is the FDA-approved drug, thymopentin (Table 1). Thymopentin binds to the active site of NSP15 endoribonuclease and may potentially block endoribonuclease activity that is responsible for the protein interference with the innate immune response. By cleaving viral RNA at uridylate, it prevents the recognition of viral RNA by the host dsRNA sensors. Thymopentin (TP5) is a synthetic pentapeptide (Arg-Lys-Asp-Val-Tyr) that belongs to the native thymic hormone thymopoietin (Goldstein and Audhya 1985). Thymopentin has been suggested for the treatment of autoimmune diseases, including chronic lymphocytic leukemia (Fan et al. 2006), rheumatoid arthritis (Sundal 1994), cancer immunodeficiency (Wolf et al. 2011), acquired immunodeficiency syndrome (AIDS) (Wolf et al. 2011), and chronic heart failure (Coppola et al. 1996). Thymopentin (Goldstein and Audhya 1985; Xiaojing et al. 2017) is also known to be an effective immunomodulatory agent that helps improve immunological condition in various patients. This repurposed drug can be used in combination with replicase inhibitors, which will inhibit the NSP15 and also improve the immunity of the patients.

The second compound, oleuropein also showed strong binding with NSP15 endoribonuclease. Oleuropein is a phenylethanoid, mainly found in the olive leaves. Oleuropein suppresses cancer cells by activating the gero-suppressor AMPK. Hence, by growth reduction in the human primary cells it leads to several transcriptomic signatures (Corominas-Faja et al. 2014). Oleuropein helps reducing colonic microflora by Hydroxytyrosol (HT). Oleuropein is known to be a potent antioxidant observed in nature to date (Sarsour et al. 2012).

The third rank compound, ginsenoside also binds tightly to the catalytic center of NSP15 structure. Ginsenosides are the natural products of steroid glycosides and triterpene saponins. Ginsenosides family consists of the oleanane family and is pentacylic in nature, composed of a five-ring carbon skeleton (Ma et al. 2020). Ginsenosides have shown diverse pharmacological and biological properties, such as antitumorigenic, anti-inflammatory, antioxidant, and inhibitor of cell apoptosis.

The top-ranking FDA-approved drug thymopentin (Goldstein and Audhya 1985; Fan et al. 2006; Wolf et al. 2011; Coppola et al. 1996; Xiaojing et al. 2017), is currently available in the market. The other two compounds, oleuropein (Corominas-Faja et al. 2014; Sarsour et al. 2012) and ginsenoside (Ma et al. 2020) are also promising ones. Hence, these drugs might help reduce the virulence of the virus and can be considered for patients being treated for COVID-19. 


\section{Conclusion}

The present coronavirus pandemic and the associated morbidity lead to the urgent need to design and develop drugs against SARS-CoV-2. In this study, structure-based virtual screening followed by the validation through molecular dynamic simulation approaches were carried out to find antiviral leads against NSP15 of SARS-CoV-2. This strategy successfully identified the FDA approved drug thymopentin, ginsenoside, and oleuropein as potential inhibitors of NSP15. The drug leads identified in this study sheds light on the pandemic infectious disease that currently lacks any specific efficient drug for the treatment. One of these drugs in combination with replicase inhibitors like remedesivir or favipiravir can prove to be a better treatment strategy for the viral infected patients. Further investigations should be carried out for these drugs to determine their efficiency that may lead to potentially therapeutic COVID-19 antivirals.

Supplementary Information The online version contains supplementary material available at https://doi.org/10.1007/s42485-021-00059-w.

Acknowledgements We thank the DPRP, Department of Science and Technology (DST), Government of India for funding. We thank DSTFIST and UGC-SAP for funding the central instrumentation facility and for extending institutional funding. We thank Pragyan Parimita Rath for carefully proofreading the manuscript.

\section{Compliance with Ethical Standards}

Conflict of interest The authors do not have any conflict of interest.

\section{References}

Bartlam MYH, Rao Z (2005) Structural insights into SARS coronavirus proteins. Curr Opin Struct Biol 15(6):664-672

Berk HCKDS, Erik L (2008) GROMACS 4: algorithms for highly efficient, load-balanced, and scalable molecular simulation. J Chem Theory Comput 4(3):435-447

Bhardwaj KPS, Alcantara JM, Yi LL, Guarino L, Sacchettini JC et al (2008) Structural and functional analyses of the severe acute respiratory syndrome coronavirus endoribonuclease Nsp15. J Biol Chem 283(6):3655-3664

Bimonte SCA, Amore A, Celentano E, Cuomo A, Cascella M (2020) Potential antiviral drugs for SARS-Cov-2 treatment: preclinical findings and ongoing clinical research. Vivo 34(3 Suppl):1597-1602

Cavanagh D (1997) Nidovirales: a new order comprising Coronaviridae and Arteriviridae. Arch Virol 142(3):629-633

Chadha SVR, Gupta S, Munde M, Gourinath S, Madhubala R (2018) Genetic manipulation of Leishmania donovani threonyl tRNA synthetase facilitates its exploration as a potential therapeutic target. PLoS Negl Trop Dis 12(6):e0006575

Chandra AGV, Qamar I, Singh N (2020) Identification of potential inhibitors of SARS-COV-2 endoribonuclease (EndoU) from
FDA approved drugs: a drug repurposing approach to find therapeutics for COVID-19. J Biomol Struct Dyn. https://doi.org/10. 1080/07391102.2020.1775127

Chikhale RVGS, Patil RB, Sinha SK, Prasad SK, Shakya A et al (2020) Sars-cov-2 host entry and replication inhibitors from Indian ginseng: an in-silico approach. J Biomol Struct Dyn. https://doi.org/10.1080/07391102.2020.1778539

Cinatl JMB, Bauer G, Chandra P, Rabenau H, Doerr HW (2003) Glycyrrhizin, an active component of liquorice roots, and replication of SARS-associated coronavirus. Lancet 361(9374):2045-2046

Coppola SBG, Laddago V, Monno L, Perrone A, Guida G et al (1996) Topical thymopentin therapy in HIV positive patients with recurrent oral candidiasis: a pilot study. New Microbiol 19(4):351-355

Corominas-Faja BSE, Cuyas E, Micol V, Joven J, Ariza X et al (2014) Computer-aided discovery of biological activity spectra for antiaging and anti-cancer olive oil oleuropeins. Aging (Albany NY) 6(9):731-741

Curreli FF-KA, Flore O (2005) Glycyrrhizic acid alters Kaposi sarcoma-associated herpesvirus latency, triggering p53-mediated apoptosis in transformed B lymphocytes. J Clin Invest 115(3):642-652

Dassault Systèmes (2016) BIOVIA discovery studio modeling environment, release 2017. Dassault Systèmes, San Diego

Deng XBS (2018) An "Old" protein with a new story: Coronavirus endoribonuclease is important for evading host antiviral defenses. Virology 517:157-163

Deng X, Hackbart M, Mattelman RC, O’Brien A, Mielech A, Yi G, Kao CC, Evans SC (2017) Coronavirus nonstructural protein 15 mediates evasion of dsRNA sensors and limits apoptosis in macrophages. Proc Natl Acad Sci USA 114(21):201618310

Dharavath SVR, Kumari K, Tomar P, Gourinath S (2020) Crystal structure of $O$-Acetylserine sulfhydralase (OASS) isoform 3 from Entamoeba histolytica: pharmacophore-based virtual screening and validation of novel inhibitors. Eur J Med Chem 192:112157. https://doi.org/10.1016/j.ejmech.2020.112157

EL Gorbalenya AE, Ziebuhr J, Snijder EJ (2006) Nidovirales: evolving the largest RNA virus genome. Virus Res 117(1):17-37

Eniyan KDS, Vijayan R, Bajpai U, Gourinath S (2018) Crystal structure of UDP-N-acetylglucosamine-enolpyruvate reductase (MurB) from Mycobacterium tuberculosis. Biochim Biophys Acta Proteins Proteom 1866(3):397-406

Fan YZCH, Yu Y, Liu J, Zhao L, Yang DJ et al (2006) Thymopentin (TP5), an immunomodulatory peptide, suppresses proliferation and induces differentiation in HL-60 cells. Biochim Biophys Acta 1763(10):1059-1066

Fehr ARCR, Perlman S (2017) Middle East Respiratory Syndrome: emergence of a pathogenic human coronavirus. Annu Rev Med 68:387-399

Forli SHR, Pique ME, Sanner MF, Goodsell DS, Olson AJ (2016) Computational protein-ligand docking and virtual drug screening with the AutoDock suite. Nat Protoc 11(5):905-919

Friesner RAMR, Repasky MP, Frye LL, Greenwood JR, Halgren TA et al (2006) Extra precision glide: docking and scoring incorporating a model of hydrophobic enclosure for protein-ligand complexes. J Med Chem 49(21):6177-6196

Goldstein GA, Audgya TK (1985) Thymopoietin to thymopentin: experimental studies. Surv Immunol Res 4:1-10

Hilgenfeld RPM (2013) From SARS to MERS: 10 years of research on highly pathogenic human coronaviruses. Antiviral Res 100(1):286-295

Jean SSLP, Hsueh PR (2020) Treatment options for COVID-19: the reality and challenges. J Microbiol Immunol Infect 53(3):436-443

Jones GWP, Glen RC, Leach AR, Taylor R (1997) Development and validation of a genetic algorithm for flexible docking. J Mol Biol 267(3):727-748 
Kang HBK, Li Y, Palaninathan S, Sacchettini J, Guarino L et al (2007) Biochemical and genetic analyses of murine hepatitis virus Nsp15 endoribonuclease. J Virol 81(24):13587-13597

Khan S, Fakhar Z, Hussain A, Ahmad A, Jairajpuri DS, Alajmi MF, Hassan MI (2020) Structure-based identification of potential SARS-CoV-2 main protease inhibitors. J Biolmol Struct Dun 1:14

Kindler EG-CC, Spanier J, Li Y, Wilhelm J, Rabouw HH et al (2017) Early endonuclease-mediated evasion of RNA sensing ensures efficient coronavirus replication. PLoS Pathog 13(2):e1006195

Kumar P, Singh A, Ngasaino MR, Shakeel I, Kumar S, Lal S, Singhal A, Sohal AA, Singh IK, Hassan MI (2020) Potential diagnostics and therapeutic approaches in COVID-19. Clin Chim Acta 510:488-497

Ma XCX, Yuan L, Wang Y, Li Z, Xu W et al (2020) Immunomodulatory effect of ginseng stem-leaf saponins and selenium on Harderian gland in immunization of chickens to Newcastle disease vaccine. Vet Immunol Immunopathol 225:110061

Mahendran GVR (2018) Neuropharmacological and molecular docking studies of xanthones from Swertia corymbosa. J Recept Signal Transduct Res 38(2):166-177

Mohammad T, Shamsi A, Anwar S, Umair M, Hussain A, Rehman MT, AiAjmi M, Islam A, Hassan MI (2020) Identification of high-affinity inhibitors of SARS-CoV-2 main protease: Towards the development of effective COVID-19 therapy. Virus Res 288:198102

Posthuma CCND, Zevenhoven-Dobbe JC, Blokhuis JH, Gorbalenya AE, Snijder EJ (2006) Site-directed mutagenesis of the Nidovirus replicative endoribonuclease NendoU exerts pleiotropic effects on the arterivirus life cycle. J Virol 80(4):1653-1661

Prentice EMJ, Lu X, Subbarao K, Denison MR (2004) Identification and characterization of severe acute respiratory syndrome coronavirus replicase proteins. J Virol 78(18):9977-9986

Quimque MTJNK, Fernandez RAT, Mendoza MAO, Liman RAD, Lim JAK et al (2020) Virtual screening-driven drug discovery of SARS-CoV2 enzyme inhibitors targeting viral attachment, replication, post-translational modification and host immunity evasion infection mechanisms. J Biomol Struct Dyn. https://doi.org/10. 1080/07391102.2020.1776639

Renxiao WLLSW (2002) Further development and validation of empirical scoring functions for structure-based binding affinity prediction. J Comput Aided Mol Des 16:11-26

Ricagno SEM, Ulferts R, Coutard B, Nurizzo D, Campanacci V et al (2006) Crystal structure and mechanistic determinants of SARS coronavirus nonstructural protein 15 define an endoribonuclease family. Proc Natl Acad Sci USA 103(32):11892-11897

Sarsour EHKM, Kalen AL, Goswami M, Buettner GR, Goswami PC (2012) MnSOD activity regulates hydroxytyrosol-induced extension of chronological lifespan. Age (Dordr) 34(1):95-109

Sawicki SGSD (2005) Coronavirus transcription: a perspective. Curr Top Microbiol Immunol 287:31-55

Schrödinger Release 2020-2 (2020a) Protein Preparation Wizard; Epik Schrödinger, LLC, New York, NY, 2016; Impact, Schrödinger, LLC, New York, NY, 2016; Prime, Schrödinger, LLC, New York

Schrödinger Release 2020-2 (2020b) Protein Preparation Wizard; Epik LigPrep. Schrödinger, LLC, New York

Sharma DPVR, Rehman SAA, Gourinath S (2018) Structural insights into the interaction of helicase and primase in Mycobacterium tuberculosis. Biochem J 475(21):3493-3509
Sinha SK, Prasad SK, Singh S, Gurav NS, Prasad RS, Gurav SS (2020a) An in-silico evaluation of different Saikosaponins for their potency against SARS-CoV-2 using NSP15 and fusion spike glycoprotein as targets. J Biomol Struct Dyn. https://doi.org/10. 1080/07391102.2020.1762741

Sinha SK, Prasad SK, Islam MA, Gurav SS, Patil RB, AlFaris NA et al (2020b) Identification of bioactive compounds from Glycyrrhiza glabra as possible inhibitor of SARS-CoV-2 spike glycoprotein and non-structural protein-15: a pharmacoinformatics study. J Biomol Struct Dyn. https://doi.org/10.1080/07391102. 2020.1779132

Slam MSSM, Akter M, Nazir K, Toniolo A, Rahman MT (2020) Coronavirus Disease 2019 (COVID-19) pandemic, lessons to be learned. J Adv Vet Anim Res 7(2):260-280

Snijder EJBP, Dobbe JC, Thiel V, Ziebuhr J, Poon LL et al (2003) Unique and conserved features of genome and proteome of SARScoronavirus, an early split-off from the coronavirus group 2 lineage. J Mol Biol 331(5):991-1004

Sundal EBD (1994) Thymopentin treatment of rheumatoid arthritis. Arzneimittelforschung 44(10):1145-1149

Vijayan RSN, Mallick BN (2007) In silico modeling of $\alpha 1 \mathrm{~A}$ adrenoceptor: interaction of its normal and mutated active sites with noradrenaline as well as its agonist and antagonist. Am J Biochem Biotechnol 3(4):216-224

Vijayan RSN, Manoharan N (2015) In silico analysis of conformational changes induced by normal and mutation of macrophage infectivity potentiator catalytic residues and its interactions with rapamycin. Interdiscip Sci 7(3):326-333

Vijayan REP, Kalaiarasan P, Subbarao N, Manoharan N (2016) Pharmacophore based virtual screening for identification of marine bioactive compounds as inhibitors against macrophage infectivity potentiator (Mip) protein of Chlamydia trachomatis. RSC Adv 6(23):18946-18957

Waziri FVR, Sahar T, Anwar S, Gourinath S, Jain SK et al (2019) In silico elucidation of novel anticancer lead molecules targeting human prostate specific gene-1 protein. J Biomol Struct Dyn 37(8):2118-2124

Wolf EMS, Boehm K, Zwahlen M, Horneber M (2011) Thymic peptides for treatment of cancer patients. Cochrane Database Syst Rev 2:CD003993

Xiaojing CYL, Yanqing G, Fangfang C (2017) Thymopentin improves cardiac function in older patients with chronic heart failure. Anatol J Cardiol 17(1):24-30

Zhang LLL, Yan L, Ming Z, Jia Z, Lou Z et al (2018) Structural and biochemical characterization of endoribonuclease Nsp15 encoded by middle east respiratory syndrome coronavirus. J Virol 92:00893-01818

Publisher's Note Springer Nature remains neutral with regard to jurisdictional claims in published maps and institutional affiliations. 